\title{
Coproduire un espace de vie urbain
}

\section{Catherine Arteau et Marie-Pascale Mignot}

\section{OpenEdition}

Journals

Édition électronique

URL : http://journals.openedition.org/communicationorganisation/2691

DOI : 10.4000/communicationorganisation.2691

ISSN : $1775-3546$

\section{Éditeur}

Presses universitaires de Bordeaux

\section{Édition imprimée}

Date de publication : 1 mai 2002

ISSN : 1168-5549

\section{Référence électronique}

Catherine Arteau et Marie-Pascale Mignot, "Coproduire un espace de vie urbain », Communication et organisation [En ligne], 21 | 2002, mis en ligne le 27 mars 2012, consulté le 19 avril 2019. URL : http:// journals.openedition.org/communicationorganisation/2691; DOI : 10.4000/ communicationorganisation.2691

Ce document a été généré automatiquement le 19 avril 2019.

(c) Presses universitaires de Bordeaux 


\title{
Coproduire un espace de vie urbain
}

\author{
Catherine Arteau et Marie-Pascale Mignot
}

1 L'opération Quartier de lumières s'est déroulée à Bordeaux, au cœur du secteur sauvegardé situé au pied de la "Grosse Cloche », édifice symbole de la forte identité historique de ce quartier médiéval alors confronté à de nouveaux enjeux économiques et urbains. Nous sommes en 1999.

2 La ville de Bordeaux ${ }^{1}$ souhaite y redynamiser les rues Saint-James, Bouquière et la place Lafargue avec la réimplantation d'activités commerciales et artisanales dans les pieds d'immeubles vacants à revaloriser. Le principe retenu: une exposition éphémère de réalisations d'artistes dans les vitrines inexploitées et une mise en place d'activités d'artisanat d'art pendant deux mois en période estivale.

3 Tenter l'expérience est pour nous l'occasion d'éprouver une croyance partagée comme architecte et comme consultant en ingénierie culturelle: l'action sur l'espace urbain trouve son envergure dans la connaissance des ressources du territoire à aménager (un site, des hommes) et parce qu'elle sait se nourrir des différentes composantes de cet « espace-temps de l'habiter " ${ }^{2}$. Écrire cette partition et l'interpréter à quatre mains devient une évidence.

4 Cette croyance est fondamentale, elle va donner au projet toute l'énergie qui est alors nécessaire face à un quartier sinistré. Très vite nous savons que cette mission va demander une implication, bien au-delà de la commande et sans laquelle l'action de communication éphémère ne pourra redonner à l'espace urbain sa force créatrice, contribuant ainsi à lui construire un avenir.

5 Il ne s'agit pas ici de proposer une modélisation de la méthode car chaque projet va naître précisément de ses particularités. En revanche, si le territoire vient alimenter le projet, cela n'est possible que dans un cadre exigeant que nous posons comme préalable à toute action. 


\section{Quartier de lumières : une opération pilote de requalification économique}

Quartier de lumières, c'est une opération pilote sur le site de la rue Saint-James dans le quartier médiéval de la ville de Bordeaux. Au cœur du secteur sauvegardé, le quartier autrefois appelé Saint-Eloi est situé entre Saint-Michel et Saint-Pierre, quartiers à forte identité. De plus, il est isolé par deux artères très passantes, les cours Victor Hugo et Alsace-Lorraine qui en font un espace traversé. Il l'est d'autant plus que la vie commerciale s'en est allée avec le départ des principaux grossistes du petit « Sentier » bordelais.

7 Dès l'appel d'offres ${ }^{3}$, nous envisageons un thème possible pour une exposition éphémère, la lumière, avec lequel il serait possible de faire intervenir des artistes, mais aussi de proposer une installation commerciale à des artisans ou commerçants sur ce thème. La lumière pourrait reprendre les réflexions du plan lumière ${ }^{4}$ de la ville de Bordeaux comme révélation des qualités urbaines et patrimoniales du site, mais aussi engendrer des interventions artistiques de concepteurs lumière dans une optique de valorisation du quartier. Déjà, nous proposons de délivrer des messages d'urbanité, d'offrir des regards et de favoriser les émotions dans un espace urbain limité et délimité par le jeu de la lumière. C'est sur cette première approche que nous avons été choisies pour mettre en place un dispositif de revalorisation du site sur une courte durée et à titre expérimental.

8 Si ce thème est une piste, il restera à vérifier sa validité après la réalisation du diagnostic et de l'étude des potentialités du quartier (enquête auprès des associations du quartier, des commerçants et des habitants...).

9 En effet, comment modifier la perception des espaces à réinvestir sans surprendre, comment imaginer une mise en scène de ces lieux sans s'imprégner d'une mémoire collective, mais également, comment se suffire d'une lecture du site ou même de son histoire sans prendre comme atout la représentation que peut s'en faire le «citoyen ordinaire »...?

\section{Des pistes de développement en appui des pôles existants}

La trame de l'événement Quartier de lumières passe alors par une véritable mise à plat de l'existant ${ }^{5}$ pour définir, dans une logique de coproduction avec les habitants, un potentiel de développement du territoire. L'objectif est de canaliser les différents signes extérieurs d'image du quartier, de les enrichir d'une double exploration (ressources du site, ressources des hommes) dans une direction favorable à l'espace concerné (dans une première phase cela concernera la rue Saint-James) et aux enjeux fixés (requalification et redynamisation).

11 Nous effectuons un repérage du site et des commerces en activité (étude quantitative et qualitative), un recensement des locaux vacants, l'analyse des potentialités et des conditions économiques de ces locaux; nous tissons des contacts et entreprenons des négociations avec les propriétaires; assurons le suivi des opportunités et la mise en relation avec des porteurs de projets d'installations commerciales, artisanales ou associatives sur le site.

12 Pour valoriser cette action il faut l'inscrire dans la réalité quotidienne du quartier: analyser les besoins réels, les demandes (comme les échanges intergénérations par 
exemple)... Ainsi nous pourrons contribuer progressivement à modifier son image jusqu'au point d'orgue: une exposition - animation éphémère, imaginée comme une remontée d'un temps historique porteur.

Ensuite seulement, nous pourrons penser à poser les bases d'un renouveau grâce à l'analyse minutieuse du terrain et à la prise en compte de la dimension humaine. Elles seules nous permettront d'imaginer des interventions durables. En effet, dans un espace aussi sinistré (un local commercial sur deux dans la rue Saint-James, voire un sur trois dans la rue Bouquière, était inoccupé) mais aussi riche de sens (logique d'identité de quartier liée à son histoire), notre conviction est assez vite la suivante : il faut s'appuyer sur les pôles déjà en fonctionnement pour explorer de nouvelles pistes de développement.

\section{L'aspect financier, un moment de confiance et de négociation}

Nous avons mené une recherche pour identifier les propriétaires de locaux commerciaux vacants, nous avons essayé de rentrer en relation avec eux (réunion avec les partenaires économiques institutionnels et contacts par téléphone). Le projet municipal de redynamisation du quartier St-James-Bouquière-Lafargue par l'économique, leurs a été présenté. Il a été plutôt bien accueilli. Les conditions de vacances et de location des magasins ont été très vite abordées. Le prix des locations de locaux commerciaux se situait dans une fourchette allant de 75 à 31 francs le $\mathrm{m}^{2}$ selon la qualité de la vitrine et des aménagements intérieurs. C'est à partir de ces données économiques que nous avons construit un scénario permettant de louer les locaux vacants à un prix attractif d'une part pour des porteurs de projets commerciaux et artisanaux (les nouveaux commerçants du quartier) et d'autre part pour les propriétaires-bailleurs (de nouveaux acteurs du quartier). Le prix de participation aux frais locatifs a été fixé à 20 francs le $\mathrm{m}^{2}$ par mois pour un bail précaire allant de quatre à six mois (incluant le temps de l'opération Quartier de lumières et les fêtes de fin d'année 1999). Les frais d'assurance du local et d'ouverture des compteurs étaient à la charge du preneur. Les bailleurs pouvaient ensuite partir sur une base plus élevée ou tout au moins négociable pour un bail commercial de longue durée. Les locaux fermés et inoccupés ont ainsi, été remis sur le marché de la location, voire de la vente. Une quarantaine de personnes le plus souvent jeunes et porteuses d'un projet de création d'entreprise ou à la recherche d'un local pour une association, ont été mises en relation avec des propriétaires-bailleurs.

La négociation n'a pas été possible avec certains propriétaires qui ont exprimé leur volonté de ne pas faire du « social » pour la ville. Ce point de vue a souvent été associé à la perte financière due à la dégradation du quartier entraînant une dévalorisation des immeubles. Certains ont noté qu'un encouragement à l'investissement dans ce quartier (incitations fiscales) n'a pas été suivi d'efforts de la collectivité localement et estiment avoir pris seuls les risques financiers. Ils ont alors souvent exprimé leur désarroi face à l'abandon d'un quartier historique par la ville de Bordeaux (taxes élevées, manque de propreté, graffitis, marginaux et insécurité...).

16 Les locaux vacants qui ont été au cœur de la commande qui nous a été faite: « réimplantation des activités commerciales et artisanales dans les pieds d'immeubles vacants » ont été l'objet d'une négociation financière d'autant moins agréable qu'elle a été revue à la baisse, mais aussi l'objet d'un gage de confiance de la part des propriétaires envers nous/médiateurs, envers la ville de Bordeaux/la collectivité et envers les porteurs 
de projets économiquement faibles. De façon surprenante et inattendue, les propriétaires-bailleurs se sont pris au jeu de la réciprocité et de la partition : chacun est acteur de la vie commune de la cité.

\section{Un projet global}

17 Ce projet économique a choisi comme terrain d'expérimentation un quartier situé au cœur du secteur sauvegardé, à proximité de la Grosse Cloche rénovée et illuminée ${ }^{6}$, qui lui confère une attractivité, notamment touristique, importante. De nombreux visiteurs se promènent dans ces rues encore pavées, lors de visites guidées ou spontanément pour photographier les monuments emblématiques du quartier (l'église Saint-Eloi ou la Grosse Cloche). De plus, sa localisation entre les cours Victor Hugo et Alsace-Lorraine, qui seront requalifiés lors des travaux du tramway (en cours), met ce quartier au cœur d'un nouvel enjeu urbain et économique.

18 Les pistes de développement pour l'opération, proposées par la ville de Bordeaux et portant sur l'aspect pittoresque et patrimonial du site (artisanat d'art et période estivale) nous sont apparues insuffisantes. En effet, les références historiques, souvent mises en exergue par le grand public comme par les élus, ne peuvent être qu'une accroche et ne doivent pas évacuer une tentative de prospective, de traitement dans une globalité urbaine, même et surtout quand il s'agit d'un site fortement marqué par l'histoire. Notre temps est alors compté (trois mois) pour imaginer et mettre en œuvre un système efficace sur un site hostile au premier abord (dégradation et paupérisation du quartier, vacances des locaux commerciaux, loyers trop élevés, problèmes sociaux et de voisinage) mais en quête d'une expression (ouverture vers une ambition).

19 «Une fabrication plus collective de la ville $»^{7}$ s'est opposée à la vision partielle voir partiale d'une opération ponctuelle dans le champ étroit de la rue - espace public à traiter en vitrine commerciale. Il s'agit bien de la rue, la rue Saint-James que nous avons choisie de traiter compte tenu des délais d'étude serrés (trois mois) et du potentiel urbain et patrimonial ${ }^{8}$ de celle-ci à entraîner l'ensemble du quartier dans une dynamique urbaine. Notre champ d'investigation s'est alors élargi. Comment mettre en relation habitants/commerçants/associations et pouvoirs publics autour d'un projet? Comment susciter de l'intérêt? Comment redonner une vie économique et culturelle à ce quartier en recherche d'une identité, d'un starter économique et d'une requalification urbaine?

La rue, cet espace public allait nous permettre de rentrer dans l'espace privé. Notre présence sur le site de plus en plus régulière et visible, les contacts avec les habitants ont permis ce « croisement d'histoires d'immeubles et d'histoires de citadins, qui peut tant apprendre sur leur constante imbrication... $»^{9}$. Au milieu des revendications de propriétaires mécontents, d'habitants inquiets et de commerçants insatisfaits, notre position d'intermédiaires entre la ville et les citadins, exclut toute démagogie et ce, malgré son caractère inconfortable. Cette neutralité est fondamentale. Elle a facilité la délivrance de messages allant bien au delà d'une animation de quartier. Il s'agissait d'une vision globale d'un quartier à reconstruire dans la ville, pour un avenir proche dans le temps et répondant aux attentes.

21 Cette vision de notre métier (faire qu'une équipe, des groupes se fixent un objectif, le définissent, s'y engagent et le réalisent) oblige, notamment pour éviter tout risque de populisme, à évoluer dans un cadre de définition de projet rigoureux. 
Quatre étapes sont nécessaires : l'observation, le tâtonnement, l'écoute, la reformulation des données pour aboutir à la formalisation du projet et/ou de l'objectif. On peut seulement alors parler de mise en œuvre: elle suppose une cinquième étape: l'accompagnement (participer à un objectif suppose un engagement constant et de réajuster ou de nourrir les motivations jusqu'à la réalisation finale) jusqu'à la fin de l'animation temporaire.

Le médiateur culturel, véritable passeur de sens est au centre de cette ambition.

\section{Saint-James, une identité retrouvée}

Une opération phare de cinq semaines, avec une mise en lumière, une exposition dans la rue, des animations culturelles et ludiques toutes les fins de semaine, s'est déroulée en septembre - octobre $1999^{10}$. La construction de ce dispositif d'animations s'est basé sur le diagnostic établi préalablement et sur une montée en puissance sur le site des acteurs du quartier accompagnés de professionnels (danseurs, comédiens, conteurs, plasticiens, graphiste, historien...).

25 L'aspect ludique sera recherché avec la construction d'événements visibles à pied ou en voiture dans la rue, ayant une portée dans le quartier et dans la ville. La hiérarchisation est souhaitée pour permettre des lectures et des moments différents. Au travers d'une exposition culturelle : l'histoire de la rue du XIVe siècle à l'arrivée du tramway en l'an 2003, racontée sur des cubes ${ }^{11} \gg$ installés au milieu de la rue durant cinq semaines, une vie de quartier s'est recréée autour d'un centre d'intérêt plus accessible avec la piétonisation de la rue et la mise en lumière de l'espace public tous les soirs jusqu'aux fêtes de fin d'année 1999. Le principe d'une installation lumière éphémère est la contribution à la construction d'un décor qui raconte l'histoire d'un quartier à reconstruire et à se réapproprier. Pour raconter Saint-James, nous avons choisi la flèche chronologique : aller d'une époque à une autre, de siècles en siècles.

6 Quartier de lumières s'est éteint le 30 octobre 1999 mais a trouvé un prolongement avec les résultats obtenus à la fin de notre mission. Il s'agit tout d'abord de locaux commerciaux vacants en juin, rouverts à l'automne (7 locaux sur le site St James-Bouquière-Lafargue dont 4 rue Saint-James et 4 projets d'installation supplémentaires en janvier 2000) ${ }^{12}$. Puis une vie de quartier en sommeil réanimée, et enfin des espoirs pour les propriétaires et les riverains, des envies et des projets pour les commerçants, des intérêts portés sur le quartier.

Cette action de "promotion » pour le quartier de la Grosse Cloche doit pouvoir poser les bases d'un avenir prometteur comme une Promesse de ville.

\section{Une action de communication pour redonner à l'espace urbain sa force créatrice}

\section{La démarche}

Que dire ? À qui ? Quoi faire ? Dans quel objectif ? Avec quelle technique? L'orientation générale, les moyens, leur gestion demandent de la rigueur, faute de quoi l'opération risque d'être inopérante. Alors comment inscrire ce qui relève avant tout d'une opération de communication dans une stratégie globale? 
Le diagnostic fait clairement apparaître un déficit d'image tel (image acquise) que nous ne pouvons pas répondre objectivement à la question «qu'est-ce que ce quartier? (image réelle). Cela va pourtant être nécessaire pour atteindre l'objectif de requalification et poser les bases d'un nouvel espace de développement économique qui s'appuiera sur les aptitudes du lieu à donner confiance, à rassurer et à se faire aimer (image désirée). Une bonne politique d'image permettra d'une part au quartier de démontrer qu'il existe, d'autre part de faire la preuve de sa valeur ajoutée et enfin qu'il peut devenir un label.

Une première nécessité s'impose, désigner le territoire qui nous occupe et qui, au-delà de son appellation administrative, a tant de mal à se nommer (quartier Saint-Eloi, quartier de la Grosse Cloche ou « entre le cours Victor Hugo et le cours Alsace-Lorraine » quand ce n'est pas le vide). Nous proposons après concertation de communiquer autour du « quartier de la Grosse Cloche ».

31 Les raisons sont les suivantes:

- historiques. L'entrée principale du bourg, à l'extrémité de la rue Saint-James, était la porte Saint-Eloi. Cette porte Saint-Eloi devint le siège de la Jurade. Et la Grosse Cloche, beffroi de la ville, fut élevée sur les deux tours construites dans le rempart intérieur qui servaient à renforcer la porte Saint-Eloi.

- touristiques. La Grosse Cloche est l'un des monuments de Bordeaux les plus visités, son attractivité est aujourd'hui renforcée par sa mise en lumière ${ }^{13}$.

- économiques. Le nom doit être porteur d'une identité qui puisse prendre le relais de l'identité de quartier artisanal puis de quartier des négociants. Il doit aller dans le sens de ce qui sera sa meilleure communication (patrimoine architectural/position en hypercentre/zone de requalification). La Grosse Cloche est porteuse de cette identité, faite d'histoire d'activités touristiques et économiques.

35 La démarche de communication vise, in fine, à doter ou modifier l'image de ce territoire. L'image n'est pas un phénomène abstrait, plaqué, mais la représentation d'un état de fait, ici paradoxal, une confrontation entre un espace urbain déstructuré, sinistré autant que riche de sens. "Apprécier, à propos de populations précises, comment les parcours migratoires ou les usages de la ville sont à la fois porteurs et signes d'acculturation, d'insertion, de socialisation, de fidélité aux origines ou encore de redéfinition des identités. Aucune action de communication urbaine ne peut passer outre cette réalité $»^{14}$ qui nous invite de ce fait à "explorer les multiples tensions qui s'instaurent entre la ségrégation et l'intégration, entre la mobilité et la sédentarité, entre la logique des liens et les pratiques des lieux $»^{15}$

6 Cette exploration nous permettra de communiquer, c'est-à-dire de bâtir pour et avec le quartier un "capital-confiance» fondé sur la valeur historique du territoire, sur sa compétence liée à sa tradition artisanale puis commerciale, son aptitude aux mutations afin qu'il puisse être choisi, apprécié, défendu (« capital-sympathie »). Cette démarche est à la fois active et réactive. Active : un parti pris d'image forte permet d'arrêter la crise et progressivement d'inverser la logique de "désamour", d'améliorer ses performances. Réactive : elle permet en outre d'éviter les faux problèmes, les rumeurs, de trouver des appuis face aux déconvenues.

Ainsi arriverons-nous à tracer un territoire personnalisé portant en lui les clés de sa réussite : nous faisons alors le pari de l'événementiel pour y parvenir. 


\section{Le pari de l'événementiel pour une stratégie de communication originale.}

l'espoir. Formes et couleurs chaleureuses imaginées comme autant de signes révélateurs de ce milieu fracturé, ces installations en traquant tous les lieux d'étranglement du sens, doivent être là comme un bilan du désenchantement ambiant autant que pour ressouder le lieu à la vie. Elles nous proposent de changer de regard. Quartier de lumières c'est pour qu'une aube de lucidité puisse se lever, pour que les yeux encore tout écarquillés comme dans un lendemain de fête, puissent regarder la réalité de ce quartier. C'est un face à face qui porte en lui la richesse ou la promesse d'une clairvoyance retrouvée. Le programme d'animation qui lui est associé a pour rôle de porter ce discours véhiculé par Quartier de lumières (révélation du territoire), de l'accompagner autant que de contribuer à lui donner vie (faire passer les spectateurs/visiteurs de l'exposition) d'une contemplation passive, désintéressée, à une participation active).

41 Seule une telle mise en scène du territoire concerné (la rue Saint-James) peut permettre de sortir des apparences (image acquise). La scène urbaine doit parler car elle est un espace actif chargé d'histoire(s), de souvenirs, de projets constituant une mémoire collective. L'empreinte de cette mémoire collective, support d'un imaginaire devient la matière première de Quartier de lumières et le creuset du changement. En effet, cet imaginaire véhiculé par une lecture, un objet, une lumière insistante... va permettre, de façon ludique, de renouveler l'utilisation des lieux, les raisons de s'y promener et les pratiques du quartier. La diversité d'expression : théâtre de rue, musique, arts plastiques, multimédia... a produit des effets renouvelés et, crescendo, une histoire, la saga d'une renaissance de quartier jusqu'à l'accomplissement d'une promesse originale de ville dont l'image authentique, séduisante, originale soit celle d'un positionnement attractif en matière économique.

\section{L'événementiel, un test grandeur nature pour accompagner les changements de la ville.}

«L'espace public et la culture urbaine dans leurs multiples expressions, constituent ces liens symboliques qui peuvent rétablir la communication entre des morceaux de ville et des groupes dissociés ou isolés $»^{17}$. L'objectif est alors de contribuer à retrouver une nouvelle formulation du rôle intégrateur de la ville, par des espaces communs réorganisés et des projets unificateurs comme Quartier de lumières : l'événementiel est une voie car il agit comme un levier sur la logique de flux des résidents et non résidents de la ville. Jusque-là nous avons expérimenté à Bordeaux la valorisation des flux :

43 - « dedans-dehors » (Fête du Fleuve);

44 - et le maillage de flux « dedans/dehors » (Fête du Vin). 
Avec Quartier de lumières, c'est le test grandeur nature d'une valorisation de flux « dehorsdedans » ou comment (re)découvrir une part de ville.

Derrière une telle démarche, c'est bien sûr la volonté d'être opérant qui préside et elle oblige à une certaine créativité. Mais l'intention va plus loin : elle pose la question de la territorialité (ici le territoire est le quartier) avec toutes les conduites d'appropriation qui lui sont assorties et de la relation à la ville. La démarche événementielle s'appuie sur cette appropriation par le citoyen (dimension affective, faire sien...). " Parallèlement à ces conduites d'appropriation, la familiarité avec les lieux suppose une installation durable mais peut aussi se nourrir ou se trouver grâce à des installations éphémères. La familiarité s'établit alors par rapport aux personnes rencontrées dans des activités (fêtes, foires, marchés...) et des lieux (habitation, travail, loisirs...), non dans un territoire ${ }^{18}$. Le partage d'espaces et de moments ordinaires (un repas, un temps lié au sport...) voire extraordinaire (les fêtes comme la Fête du Vin à Bordeaux), la coexistence. « la sociabilité sans engagement particulier sont autant de comportements urbains qui contribuent à décloisonner la notion de quartier au profit de la ville $»^{19}$. Les espaces ne sont pas seuls en cause, mais ils s'insèrent dans la trame des événements et des relations, apportent leur tonalité propre.

Cette conception de l'organisation de la ville exclut toute hiérarchie a priori puisqu'elle s'appuie sur une trame (un maillage de l'espace urbain). On voit bien alors que la notion de quartier ne peut être réduite à un fragment plus ou moins agréé par la ville.

L'événementiel par son caractère inédit et dynamique est un outil pour une meilleure adaptabilité de la ville, de ses habitants aux changements les plus simples (l'arrivée d'un nouveau lieu de vie) aux plus spécifiques (la requalification) comme aux plus ambitieux (projet urbain)... «Cette reconnaissance des capacités des citadins à la coproduction d'espaces est encore peu fréquente $»^{20}$ même chez les concepteurs (architectes, urbanistes...). Certains cependant refusent la logique de moules dans lesquels les habitants devraient trouver leur place.

\section{Décloisonner la notion de quartier au profit de la ville}

\section{« Dedans-dehors », un aller-retour riche de sens et de liens avec la ville}

L'opération de type événementiel a apporté un éclairage sur le fonctionnement urbain du quartier, notamment sur les extensions possibles vers d'autres quartiers ou vers d'autres pôles de la ville. Redonner une dynamique commerciale à la rue Saint-James (opération Quartier de lumières) dans un premier temps, avec une poursuite des efforts engagés par tous (investissement de la ville, des propriétaires, des commerçants et de partenaires), mettre en œuvre un projet sur la rue Bouquière ${ }^{21}$ (projet de développement autour du multimédia) et sur la place Lafargue (projet de parking souterrain de la Communauté Urbaine de Bordeaux) toujours en prise avec les données du terrain et réaliser la proposition du «souk littéraire ${ }^{22}$ pour le quartier à l'échelle de la ville. Repousser les limites du quartier sans cesse du dedans (« on est bien dans le quartier »; les ressources) au dehors et du dehors au dedans (" amener les bordelais à nouveau dans le quartier ») ; faire partie de la ville et du projet urbain mis en œuvre par la ville de Bordeaux) pour trouver la juste mesure. Tout plan d'aménagement urbain aurait pu tracer des extensions 
possibles ou des liaisons urbaines, mais ici c'est l'expérimentation de la coproduction qui a permis de révéler les extensions spatiales certaines. En effet l'espace est instable, imperceptible sur un plan, mais bien réel dans le lien social qu'il établit, dans l'image qu'il façonne, dans la régénération urbaine ${ }^{23}$ qui relie les quartiers à la dynamique urbaine.

\section{La recherche d'un équilibre durable vers une promesse de ville}

Après une première mission menée de mai à fin octobre 1999, portant à la fois sur la connaissance du terrain (inventaires, contacts personnalisés...) et sur la mise en place de l'opération Quartier de lumières (exposition éphémère, installation lumière et animations), nous pensons, au regard des premiers résultats, que le site Saint-James, Bouquière et Lafargue aurait $\mathrm{pu}$ heureusement bénéficier d'un accompagnement pour son redéveloppement économique, urbanistique et pour son positionnement identitaire. En effet, nos hypothèses présentées en août 1999 s'accompagnent d'un projet fédérateur et cohérent qui s'inscrit dans la durée ou l'inscription d'un projet global sur le site SaintJames/Bouquière/Lafargue (Promesse de ville):

51 - avec la mise en réseau et la prise en compte des talents et des énergies dans le quartier (commerçants, riverains, mais aussi passants...) ;

52 - avec un programme de réhabilitation des immeubles intégrant cette logique de coproduction ;

- avec l'ensemble valorisé par un projet de communication conséquent sur le fond et grâce à une planification dans le temps.

Seul un vrai projet organisé comme tel et porté par une volonté politique permet d'enraciner ce qui a été amorcé. L'idée de l'accompagnement du quartier jusqu'à une maturité urbaine (souhaitée) rejoint l'actualité du développement durable. Si celui-ci concerne surtout l'environnement, nous faisons un parallèle à l'échelle de l'urbain car les ressources sont identiques et les objectifs similaires. Il s'agit pour la ville de Bordeaux de « préserver la mixité sociale, promouvoir une ville et une vie de proximité... ». Très tôt dans notre démarche, nous avons senti l'enjeu d'un équilibre durable à travers le projet Promesse de ville, construite comme une promesse mutuelle et bilatérale entre la collectivité et le citadin.

Ne s'agit-il pas de trouver une conciliation entre ressource du site et des hommes et le développement urbain. Comment imaginer qu'une opération même fondée tant en intentions qu'en résultats, puisse inverser une tendance? Aujourd'hui les commerces « vont et viennent » et l'exploitation du site n'est pas au bout de ses possibilités. Ce bout de ville attend son heure pour l'aménagement de l'espace public, la mixité sociale dans l'habitat, l'implantation de parkings... et quelle valorisation possible d'une opération pilote comme Quartier de lumières si décideurs, médiateurs, aménageurs, ne travaillent pas ensemble à construire la ville, celle de l'échange, du tissage, du maillage... soutenus. Daniel Cohen ${ }^{24}$, économiste, relève le manque de corps intermédiaires : «il ne suffit pas de casser des vieux immeubles; il faut relier les gens à la vraie ville, celle qui vit... ». Nous avons fait l'expérience du lien social de la ville qui unit, de l'expression sociale mise au profit de la coproduction d'un moment de vie urbaine, d'abord avec l'opération Quartier de lumières puis en imaginant Promesse de ville qui en devient son possible prolongement sur la durée, le « souk littéraire » étant une voie. 


\section{« Restituer aux habitants une participation à la vie de la cité et l'appropriation de leur vie de citadins $»^{25}$}

56 L'analyse du discours d'habitants auxquels des anciens vont proposer un jeu de photographies montrant des espaces connus ou inconnus, soignés ou dégradés... va permettre, derrière l'anecdote, de mettre à jour les structures d'un discours collectif sur les images du quartier et du quartier dans la ville

57 L'idée est ici de partir de l'expérience des habitants pour comprendre l'image du territoire (l'espace seul n'explique rien) et par là même son aptitude à toucher, faire comprendre, donner envie... Ces images du quartier dans la ville perçues par les personnes interrogées construisent des images collectives de la ville et, sur un autre registre, un lien intergénérationnel, un lien social...

58 «Le droit à la ville, c'est le droit à la vie urbaine transformée, renouvelée, qui sans abandonner les acquis de la modernité - la croissance, l'individualisation, les loisirs maintiendrait en les transformant, les formes de la centralité, le droit à l'œuvre (l'activité participante), et le droit à l'appropriation (distinct du droit de propriété) $»{ }^{26}$

\section{Pourquoi «Quartier de lumières » fait aujourd'hui référence?}

Les résultats encourageants de l'opération ne sont pas la seule raison de l'intérêt porté à notre travail par des professionnels, notamment de l'urbanisme. Il a d'abord surpris :

- travailler en transversal des organisations est inhabituel ;

61 - donner un rôle fondateur à un outil éphémère : l'événementiel peut paraître paradoxal.

62 Une telle démarche n'avait pas beaucoup de chance de convaincre a priori. Mais parce qu'elle a fait la preuve du caractère privilégié de l'événement pour mobiliser les habitants et les acteurs institutionnels et économiques du territoire sur lequel ils se déroulent, elle a pris un caractère exemplaire ${ }^{27}$. Nous souhaitons donc conclure sur cette ouverture que représente la dimension événementielle : accompagner le changement en offrant une occasion exceptionnelle de programmer et d'engager des projets de grande ampleur ou de leur associer des initiatives complémentaires (et nécessaires) à l'équilibre général qui marquent durablement leur ville. L'impact d'une telle approche (prenons Quartier de lumières comme exemple) pourra s'exercer dans de multiples domaines, en termes :

63 - d'image interne et d'identité : contexte de morosité et de scepticisme, redonner confiance aux habitants, les rassembler toutes origines sociologiques confondues et quels que soient leurs clivages.

64 - d'aménagement urbain: stratégie de reconversion d'une zone urbaine dégradée (restructuration de la voirie, remise en état de sites, acquisitions foncières, requalification de l'environnement).

65 - d'environnement : inversion des modes de consommation de l'espace public, la logique "paupérisation/dégradation» fait progressivement place à la logique "valorisation/ création » fondatrice pour la requalification.

66 - de développement économique et touristique: effet de levier, dynamisation de l'immobilier, lisibilité et accessibilité du patrimoine 
67 - de finances publiques par un déplacement potentiel des affectations budgétaires qui passent de la fonction « réparation » à la fonction « construction ».

Mais aussi, en raison des processus qu'il aura fallu mettre en œuvre pour leur préparation et leur déroulement, ils peuvent influer sur les acteurs eux-mêmes, sur la population, comme sur les modes de gestion de la cité.

Ce type d'organisation autour d'un événement met en œuvre des méthodes et des savoirfaire très différents de ceux demandés pour l'administration au quotidien d'une métropole. Elle requiert la mise en place de formes de coopérations inédites entre les différentes autorités publiques et les acteurs privés, qui peuvent aller effectivement jusqu'à changer les méthodes d'approche de la ville (remise en cause de la routine). À l'intérieur des institutions, elle entraine des évolutions dans la gestion des ressources humaines, pour permettre aux individus de mieux utiliser les capacités d'initiative, d'autonomie et d'enthousiasme nécessaires au $\operatorname{projet}^{28}$. Au niveau de la société dans son ensemble, la préparation d'une telle organisation et sa réalisation ont des effets dynamisants sur la population.

Mais ces changements importants peuvent cependant s'estomper rapidement et doivent s'inscrire dans une logique politique où le changement devient un facteur de créativité.

71 Enfin, une telle opération donne au mot communication toute son envergure : en amont elle se nourrit de l'échange ( transmettre ", « être en rapport $»^{29}$ ), en bout de ligne elle génère des opportunités de communication («faire partager », « être en relation $»^{30}$ ). Elle trouve son fondement comme sa légitimité à travers le nécessaire rôle intégrateur de la ville (lettres de noblesse de la gestion de la cité) et permet cette rencontre entre espace et communication.

La médiation culturelle est au cœur de cette interaction entre les populations et leur espace de vie dans toutes ses composantes en permanence traversées par le changement. Les villes sont désormais "le monde naturel $»^{31}$ «de l'homme et l'urbanisme doit concilier «la rigueur du plan et des normes de la vie urbaine avec les possibilités d'expression de la subjectivité $\aleph^{32}$. Le rôle du médiateur culturel est de faire le lien entre subjectivité et système objectif. Il est un "capital-risqueur » au centre des politiques de la ville (politique de la ville qui sous-entend un champ d'intervention en transversal).

La ville moderne que nous décrivons, nous l'avons expérimentée en 1999 rue Saint-James dans cette coproduction d'un espace de vie urbain mais seul l'accompagnement, bien évidemment porté par une vision politique, permet de développer une culture moderne de ville ${ }^{33}$.

BIBLIOGRAPHIE

\section{Ouvrages}

CLAVEL, M., Sociologie de l'urbain. Paris : Anthropos-Economica, 2002.123 p.

GRAFMEYER, Y., Sociologie urbaine. Paris : Nathan-Université. 1999. 128 p. 
LEVÊQUE, C, Plus de lumière. Galerie du jour - agnès b. villa arson, 1998. LUSSAULT, M., BODYGENDROT. S., sous la direction de T. PAQUOT. La ville et l'urbain, série l'état des savoirs. Paris :

Éditions la découverte, 2000. 443 p.

Evénements, tourisme et loisirs. Les Cahiers Espaces. n 74, août 2002.175 p.

WESTPHALEN, M. H, Communicator. 3è édition. Paris : Dunod 2000. 420 p.

\section{Revues}

Développement durable : l'enjeu urbain : dossier. Revue Urbanisme, mai-juin 2002, n 324, p. 47-51

Régénération urbaine en Europe. Revue Urbanisme, mars-avril 2002, n 16, Hors-série

Ouvrages divers

Plaquette de présentation : Projet urbain pour la ville de Bordeaux - Mairie de Bordeaux, octobre 1996.

Travaux d'étudiants : BOUSSAIS Juliette, COUTURIER Mathilde, GÂTÉ Yves, MOREAU Florence, Plan de communication - Opération Centre 2000 - Quartier Saint-Eloi. DESS option A, ISIC, Université Michel-de-Montaigne Bordeaux 3, juillet 1999

Livret d'exposition : Quartier de Lumières. La rue Saint-James sous toutes ses facettes, juin-octobre 1999

\section{NOTES}

1. À l'initiative de la Direction du Développement Economique de la ville de Bordeaux, le projet a trouvé le soutien financier de la Communauté Urbaine de Bordeaux et du Ministère de l'Artisanat et du Commerce. La Chambre de Commerce et d'Industrie de Bordeaux et la Chambre des Métiers de la Gironde ont été les partenaires techniques de l'opération.

2. Maïté Clavel. Sociologie de l'urbain. Anthropos-Economica, 2002, p. 21

3. Notre mission comprenait aussi la gestion de l'enveloppe budgétaire qui s'est élevée à 350000

F. toutes taxes comprises pour l'ensemble du dispositif Quartier de lumières (honoraires des chargées d'étude, prestations de plasticiens et graphiste, cachets d'artistes et de comédiens, divers matériels et prestations techniques sur le site, publicité).

4. Plan lumière conçu par l'agence Concepto - Roger Narboni concepteur-lumière, en 1996 pour la Ville de Bordeaux.

5. Cet " entre-deux », entre Saint-Pierre et Saint-Michel, quartiers bien identifiés dans la ville, confère à ce quartier un manque d'identité, ressenti par les commerçants et par les habitants selon l'enquête réalisée par les étudiants en DESS Communication des Organisations à l'I.S.I.C. (Institut des Sciences de l'Information et de la Communication) de l'Université Bordeaux 3 : Juliette, Mathilde, Laurence, Yves, juillet 1999.

6. Mise en lumière du monument la Grosse Cloche par l'architecte Christine Matthieu.

7. Jean-Pierre Frey. Urbanisation et habitat. In La ville et l'urbain, série L'étal des savoirs. Paris : éditions La découverte, 2000. p. 189.

8. Ses atouts visibles étaient : la présence de la Grosse Cloche, monument emblématique connu de tous, la déclivité du sol entraînant un effet de perspective fort vers la place Lafargue au point bas et vers la Grosse Cloche au point haut, la présence de témoignages historiques et architecturaux, la fréquentation du public.

9. Yves Grafmeyer, Peuplement et mobilités. In Sociologie urbaine. Paris : Nathan-Université, 1999. p. 62 . 
10. Quartier de lumières - La rue Saint-James sous toutes ses facettes, livret de l'exposition où les artistes et concepteurs de l'opération se retrouveront.

11. Dix cubes de $1,20 \mathrm{~m}$ de côté, sur roulettes. La journée de 11 heures à 23 heures, ils étaient placés au milieu de la rue. La nuit, ils étaient garés comme des voitures le long du trottoir.

12. Pour les fêtes de fin d'année, l'école des Beaux-arts de Bordeaux a ouvert le show-room de ses étudiants.

13. Ce thème de la lumière qui deviendra notre fil conducteur pour l'exposition éphémère est plus qu'une intention, il est à ce point essentiel à la pensée médiévale qu'on le retrouve pour ainsi dire partout (métaphore et modèle).

14. Maïté Clavel. ibid. p. 107.

15. Maïté Clavel, ibid. p. 107.

16. Char René, Le Nu perdu. 1972.

17. Maïté Clavel, ibid. p. 87.

18. Maïté Clavel. ibid. p. 78.

19. Maïté Clavel, ibid., p. 78.

20. Maïté Clavel. ibid., p. 45.

21. Un travail identique dans la méthode sur la rue Bouquière pourrait être une suite donnée à notre mission avec cependant une spécificité urbaine encore différente.

22. Le souk littéraire est une proposition de rencontre destinée aux jeunes créateurs d'entreprises. Ce souk situé entre le pôle économique lié au multimédia et le pôle des libraires de la ville s'ouvre à toute activité qui fait le lien entre les savoir-faire traditionnels comme l'imprimerie (les Essais de Montaigne ont été imprimés rue Saint-James) et les nouvelles technologies sous les formes les plus diverses (du vrac de pièces au tri du matériel périmé...). Durée de deux ans. cadre juridique et financier valorisant (accompagnement, résidence de créateurs...).

23. Dans le cadre d'un groupe de travail sur la régénération urbaine dans l'Europe du NordOuest, six villes: Lille Métropole, Valenciennes, Manchester. Birmingham. Rotterdam et Bruxelles ont approfondi les thèmes de la participation des habitants, du rôle des investisseurs privés, de la relation entre régénération urbaine et aménagement du territoire, des échelles territoriales d'intervention.

24. Journal Libération. Interview de Daniel Cohen par Hervé Nathan. 18-19 mai 2002. p. 5.

25. Maïté Clavel. ibid.. p. 19.

26. Maïté Clavel. ibid., p. 21.

27. Une journée consacrée au thème "Changer la ville» a présenté l'opération Quartier de lumières à Lille en novembre 2001 durant le forum de la Communication publique (CAP'COM).

28. A ce titre, il faut citer l'exemplarité de l'intervention des services techniques de la ville de Bordeaux.

29. Extrait de la définition du mot cité dans le dictionnaire Larousse.

30. Extrait de la définition du mot cité dans le dictionnaire Larousse.

31. Maïté Clavel, ibid., p. 17.

32. Maïé Clavel, ibid., p. 17.

33. Donner une suite à Quartier de lumières s'inscrirait dans cette modernité. 


\section{RÉSUMÉS}

Une opération pilote Quartier de lumières pour un secteur sauvegardé à Bordeaux. Les enjeux : redynamiser cet espace urbain, poser les bases d'une requalification économique. Les atouts : un quartier à forte identité, la mise en lumière de la "Grosse Cloche ", l'arrivée du tramway. Les limites : un quartier isolé, un espace traversé, le départ des principaux acteurs économiques, la paupérisation. La méthode : une action de communication pour redonner à l'espace urbain sa force créatrice. Le résultat: l'opération " Promesses de ville " pour asseoir la légitimité de cet espace urbain, et reconquérir durablement sa place dans la cité.

À pilot project for the urban renewal of an historical venter of Bordeaux. What is at stake : reviving this urban area. laying the foundations for the reinstatement of its role as a key district in the City 's economy. Winning cards : the district 's strong identity. the lighting enhancing the "Grosse Cloche». the newly installed tramway. Limits: the district isolation, its «thoroughfare "aspect, the delocation of its chief économie players, pauperization. Means : a communications project aimed al stimulating créative enterprises in this district. Result: operation « Promesses de ville» in order to religitimize this urban area. and durably reestablish its importance as one of the foremost assets of the city of Bordeaux.

\section{INDEX}

Mots-clés : médiation culturelle, redynamisation urbaine, lien social, centre urbain ancien, mutations urbaines

\section{AUTEURS}

\section{CATHERINE ARTEAU}

Catherine Arteau est historienne de l'Art, certifiée d'épigraphie et de paléographie arabes, diplômée de muséologie, consultant en ingénierie culturelle et touristique. Dans sa pratique professionnelle, elle développe le concept d'élite nombreuse » et amène la médiation culturelle au cœur des enjeux de la ville. Membre adhérent de la Société Française du Coaching. elle intervient en tant que coach dans le domaine du management de projet, de l'animation et de la motivation des équipes ou des groupes. Tél.

0143316582

\section{MARIE-PASCALE MIGNOT}

Marie-Pascale Mignot. architecte D.P.L.G. et maître de conférence associée à l'I.S.I.C. (Institut des Sciences de l'Information et de la Communication) Université Bordeaux 3, développe le rapport entre espace et communication, au sein de l'université et dans sa pratique professionnelle avec le réseau de professionnels Citadin-e-s (management 
urbain, médiation culturelle, mise en visite du patrimoine, muséographie, scénographie d'événements et d'expositions, signalétique). Tél. 05579578 